\title{
CAPACIDAD DE CAPTURA DE CARBONO DE LA PALMERA DATILERA Phoenix dactylifera COMO SERVICIO AMBIENTAL EN EL DISTRITO DE TACNA
}

CARBON CAPTURE CAPACITY OF THE PALMERA DATILERA Phoenix dactylifera AS AN ENVIRONMENTAL SERVICE IN THE DISTRICT OF TACNA)"

Fernando Edward Loyola Cabana ${ }^{1}$

Richard Sabino Lazo Ramos ${ }^{1,2}$

Información del artículo: Recibido: 18/08/2017. Aceptado: 17/09/2017

1 Ingeniero Ambiental 2 Doctor, docente de la Facultad de Ingeniería, Universidad Privada de Tacna 
Julio - Diciembre del 2019. 


\section{Resumen}

En los últimos años, el parque automotor en la ciudad de Tacna ha aumentado, así como la emisión de gases contaminantes tales como el dióxido de carbono (CO2) generados por diferentes tipos de vehículos principalmente los del transporte público en la ciudad de Tacna. EI trabajo de investigación tuvo como objetivo determinar la captura de carbono como servicio ambiental mediante las palmeras que se encuentran en la avenida Bolognesi, avenida Industrial y alrededores. Para ello, se utilizó el documento Identificación de metodologías existentes para determinar stock de carbono en ecosistemas forestales del Ministerio del Ambiente (MINAM), se optó por estimación de carbono en la biomasa arbórea a través del método no destructivo directo que consiste en la estimación de la biomasa con datos dasométricos los cuales fueron tomados en campo. El estudio se desarrolló entre los meses de junio a octubre del 2016, en la cual se determinaron dos estratos de palmeras que están expuestas a las emisiones de CO 2 atmosférico proveniente de vehículos ligeros, estableciéndose ocho puntos de muestreo por zona, en la cual, se tomaron un total de 16 muestras. Los análisis de las muestras se realizaron durante los meses posteriores a octubre donde concluyó la toma de datos. Los resultados fueron los siguientes: La zona 1 ubicada alrededor de la Avenida Bolognesi con 637 individuos de Phoenix dactylifera tuvieron un contenido de 136,87 Tn CO2e y para la zona 2 ubicada alrededor de la Avenida Industrial con 369 individuos de Phoenix dactylifera tuvieron un contenido de 18,35 Tn CO2e, donde la zona 1 tendría mayor cantidad de CO2 almacenado debido a tener alrededor del $60 \%$ de la población total de los individuos. Finalmente se puede comprobar que la capacidad de captura de carbono (CO2) de la Phoenix dactylifera actúa como servicio ambiental en el distrito de Tacna, aportando de forma positiva a la calidad de aire, como condición fundamental para el desarrollo de la vida de las plantas y como mitigación al cambio climático por emisiones de $\mathrm{CO} 2$ como gas de efecto invernadero, el cual proviene del parque automotor de Tacna.

Palabras Clave: Carbono, Phoenix dactylifera.

\section{Abstract}

In recent years, the car park in Tacna' city has increased, as well as the emission of polluting gases such as carbon dioxide (CO2) generated by different types of vehicles, mainly those of public transport in the city of Tacna. The research work was aimed at determining the carbon capture as an environmental service through the palm trees that are in the Bolognesi avenue, Industrial avenue and surroundings. For this purpose, the document Identification of existing methodologies to determine carbon stock in forest ecosystems of the Environment's Ministry (MINAM) was used. Carbon estimation in tree biomass was chosen using the non - destructive direct method, which consists of estimating of the biomass with dasometric data which were taken in the field. The study was carried out between June and October of 2016, in which two strata of palm trees were determined that are exposed to atmospheric $\mathrm{CO} 2$ emissions from light vehicles, establishing eight sampling points for zone, in which, took a total of 16 samples. Samples were analyzed during the months after October, when data collection was completed. The results were as follows: zone 1 located around Bolognesi avenue with 637 specimens of Phoenix dactylifera had a content of 136,87 Tn CO2e and for zone 2 located around Industrial avenue with 369 specimens of Phoenix dactylifera had a content of 18,35 Tn CO2e, where zone 1 would have the largest amount of $\mathrm{CO} 2$ stored due to having about $60 \%$ of the total population of the individuals. Finally, it can be verified that the carbon capture capacity (CO2) of the Phoenix dactylifera acts as an environmental service in the district of Tacna, contributing positively to air quality, as a fundamental condition for the development of plant life And as mitigation to climate change by emissions of $\mathrm{CO} 2$ as a greenhouse gas, which comes from the car park of Tacna.

Keywords: Carbon, Phoenix dactylifera 


\section{INTRODUCCIÓN}

Cuando la composición del aire cumple con los porcentajes de existencia de los diferentes gases que lo conforman, se habla del concepto de "aire puro" y que en esta circunstancia la atmósfera terrestre se encuentra en el llamado "equilibrio ecológico". Debido al incremento de la emisión de gases productos de las actividades antropogénicas y de los gases de efecto invernadero (GEI), ha ocasionado que la humanidad adquiera una mayor conciencia del rol que está desempeñando en el incremento de éste fenómeno natural. Actualmente en el Perú, en materia ambiental se ha avanzado progresivamente teniendo a nuestra disposición los Límites Máximos Permisibles (LMP's) y Estándar de Calidad Ambiental (ECA's) para Aire en este caso, que son importantes para garantizar el derecho de un ambiente sano y buena calidad de vida. En Tacna, por otro lado, el parque automotor se ha incrementado en los últimos años, desde 40421 vehículos registrados en el 2005 a 47900 vehículos registrados a finales del 2015 aproximadamente según la sub gerencia de transportes de la Municipalidad Provincial de Tacna (MPT).En el trabajo se realiza la determinación de la capacidad de captura de carbono de la especie Phoenix dactylifera como servicio ambiental en el distrito de Tacna, especie que se encuentra ampliamente distribuida por el mundo. La Phoenix dactylifera tiene importancia como especie introducida y en el Perú, la distribución es amplia por toda nuestra costa, donde su principal rol ha sido de relevancia ornamental. En Tacna también ha cumplió ese rol ornamental y está distribuida en el cercado del distrito. En la ciudad de Tacna, puntualmente en el cercado del distrito, la presencia de emisiones de $\mathrm{CO} 2, \mathrm{CO}$, entre otros gases producto de la combustión interna de vehículos automotores se evidencia, debido a las actividades diarias de transporte público y servicio particular. Los gases generan incidencia en el medio ambiente circundante de la comunidad tacneña producto del incremento de $\mathrm{CO} 2$ del parque automotor. Indica Gracia (2006), en Indicadores ambientales y Paisajísticos del Palmeral de ELCHE explica que estudió 35 ha en las cuales estaba presente la especie Phoenix dactylifera de aproximadamente 50 años de las cuales. De acuerdo con Gonzáles (2001), en las palmas de Gran Canaria, estimó niveles y estructuración genética entre poblaciones de la especie endémica de Islas Canarias ( $P$. canariensis), comparándola con su congénere ampliamente distribuida Phoenix dactylifera, suponía el $75 \%$ del total de la variación genética detectada en P. canariensis separan claramente las poblaciones de P. canariensis y P. dactylifera. Rodríguez, Jiménez, Meza, Aguirre, \& Razo Zarate (2007), realizó estimación de carbono en el bosque tropical y localizó en el componente aéreo de fustes, ramas y hojas con 68,4 ton $C$ ha- 1 y el menor contenido fue la necromasa con solo 0,7 ton C ha-1. Cabrera \& Wallace (2007), generó datos de densidad y patrón de distribución espacial de palmeras arborescentes en bosque andino-amazónico en Bolivia, y encontró en 10,28 ha evaluadas, Socratea exorrhiza (47,1 individuos/ha) e Iriartea deltoidea (45,8 individuos/ha). Reyes \& Gutiérrez (2010), describe el cuidado, manejo y disposición de los árboles que condicionan los servicios ambientales aprovechables en el contexto urbano. Londoño, Correa, \& Palacio (2011) realiza la estimación de emisiones de contaminantes atmosféricos provenientes de fuentes móviles en el área urbana de Envigado. Navarro, Díez, \& Meléndez (2013), en el palmeral de Elche, estimó la cantidad de carbono y nitrógeno que permanece retenida en el palmeral patrimonio de la humanidad. Para Castro \& Escobar (2006), la estimación de las emisiones contaminantes por 
fuentes móviles a nivel nacional y formulación de lineamientos técnicos para el ajuste de las normas de emisión, sirvió en los niveles de emisiones en caliente de CO, HC, NOx, MP. En Perú, Malca (2001), en Estimación de la capacidad de captura de CO2 en bosques secundarios del trópico Amazónico como indicador de Valorización Económica, indicó la capacidad de captura en la selva baja situada por debajo de los 400 msnm. Catpo (2004), calculó la cantidad total de biomasa con el número de árboles en las 196 ha, finalmente el valor hallado sé multiplicó por el factor de conversión de carbono $(0,5)$ obteniendo el carbono en las plantaciones en t/ha.Palomino (2007), realizó la estimación del servicio ambiental de captura del CO2 en la flora de Los Humedales de Puerto Viejo. Cobos (2012cuantificó el carbono almacenado en las especies forestales maderables del área calculando biomasa aérea. Valera (2013), mide la influencia de condiciones climáticas en la captura de carbono en un sistema Theobroma sp "cacao". Pari (2013), estima la cantidad de carbono por hectárea de plantaciones y rebrotes de Eucalyptus globulus Labill. Castillo (2014), sugiere que el uso de combustibles limpios y la modernización del parque automotor lograrán disminuir la contaminación del aire además de la forestación. Valenzuela (2015), indica la diferencia del aporte de entre la biomasa viva sobre el suelo (vegetación arbórea y vegetación no arbórea) y la biomasa muerta (detritos y hojarasca), siendo la biomasa viva sobre el suelo, mayor. En Tacna, Pari (2009), refiere que la influencia del estado de vehículos gasolineros y diesel de transporte masivo urbano y los gases contaminantes vehiculares en Tacna estuvo sujeta a las revisiones técnicas satisfactorias. Pancca (2014) encuentra valores de material articulado de $2,5(\mathrm{ug} / \mathrm{m} 3)$ que sobrepasan los estándares de calidad ambiental (ECA), en los distritos de Alto de la Alianza (ISTP Francisco de Paula Gonzáles Vigil - Av. Jorge Basadre), Ciudad Nueva (Centro de Salud Ciudad Nueva-Calle Daniel Alcides Carrión) y Tacna (Gran Hotel Tacna-Av. Bolognesi). Aranibar (2014), indica el estado del bosque de Polylepis y su eficiencia en la Captura de CO2 en Tarata.

Dentro del estudio del aire y su composición, existen parámetros que se consideran en función a los niveles de concentración de los mismos y denominados "contaminantes del aire" y son considerados estándares primarios (Castillo, 2014). En la troposfera una masa de aire puede dar la vuelta a la Tierra en pocos días (Rivera, 2012). Las fuentes antropogénicas son la principal fuente de emisión de $\mathrm{CO} 2$ actual (Panel Intergubernamental de Cambio Climático IPCC, 2005). En la ciudad de Tacna también se realiza la explotación de combustibles fósiles para el desarrollo de diversas actividades productivas (fuentes fijas) y las actividades de transporte (fuentes móviles).

El carbono es un componente esencial para los seres vivos existe en su mayor parte como dióxido de carbono en la atmósfera, los océanos y los combustibles fósiles (carbón petróleo y otros hidrocarburos). Los bosques, en particular, juegan un papel preponderante en el ciclo global del carbono $(C)$ debido a que almacenan grandes cantidades de carbono en su biomasa, intercambian carbono en la atmósfera a través de la fotosíntesis. (Gonzáles, 2001). En la medida que el árbol crece va incrementando su follaje, y todos los componentes de la copa aportan materia orgánica al suelo, que al degradarse e incorporarse da origen al humus estable, éste, a su vez, aporta nuevamente CO2 al entorno (García, 2011). Para determinar la cantidad de carbono que un bosque secuestra, almacena o libera, es necesario identificar y analizar cada fuente carbonada que existe en la vegetación, tanto en sus componentes aéreos y subterráneos, así como también en el suelo (necromasa y contenido mineral). 


\section{OBJETIVOS}

- Estimar la cantidad de carbono capturado y almacenado en la biomasa arbórea aérea de Phoenix dactylifera como CO2e.

- Correlacionar el carbono capturado por Phoenix dactylifera con su crecimiento producto de las emisiones de CO2e presente en el distrito de Tacna.

\section{METODOLOGÍA}

Investigación descriptiva correlacional para cuantificar el carbono almacenado a través de los elementos dasométricos medibles (DAP y Altura) de la especie Phoenix dactylifera. El ámbito y tiempo de la investigación comprendió el distrito de Tacna: Zona I: Cuadrante comprendido por la Av. Leguía, Av. Basadre y Forero con Av. Circunvalación-Colpa y Av. Bohemia tacneña y Zona II: Cuadrante comprendido por la Av. Leguía, Av. Basadre y Forero con Av. Industrial y Av. Jorge Basadre. El tiempo de la investigación corresponde al año 2016, durante los meses de mayo a octubre. La población de estudio estuvo conformada por la distribución de la especie Phoenix dactylifera en las zonas I y II las cuales fueron estratificadas en (Estrato I) con $\mathrm{H}$ (altura) < $10 \mathrm{~m}$. y (Estrato II) con $\mathrm{H}$ (altura) $>10 \mathrm{~m}$. comprendidas aproximadamente en un área total de 1000 ha en el distrito de Tacna.Se evaluó 69 palmeras de un total de 1006 palmeras distribuidas en el distrito (7). La identificación de la especie se georeferenció con sistema de coordenadas universal transversal de Mercator (UTM). Se utilizaron los siguientes instrumentos: Clinómetro, Cinta métrica de $3 \mathrm{~m}$, Cinta métrica Stanley de $30 \mathrm{~m}$, formato de recolección de ángulos y circunferencia y formato de conversión de Altura y DAP. Para obtener los datos dasométricos se recopiló información de inclinación (clinómetro), distancia (cinta métrica de $30 \mathrm{~m}$.), la longitud de la circunferencia del estipe (cinta métrica de $3 \mathrm{~m}$.), y mediante conversión dieron los datos dasométricos (altura y Dap) para la cuantificación de biomasa. Se consideró una serie de fórmulas y factores que se basaron en los estudios preliminares y de las guías elaboradas en el Perú relacionados a la cuantificación de carbono como medio de mitigación al cambio climático. Para la cuantificación de depósitos de carbono a medir se tomó como referencia del Centro Internacional para la investigación en Agroforestería - ICRAF y de la Guía "Identificación de Metodologías existentes para determinar stock de carbono en ecosistemas forestales" del MINAM. Para la determinación del contenido de carbono en la especie Phoenix dactylifera se seleccionó sub metodología para la estimación de solo la biomasa arbórea aérea, la cuantificación total y su equivalente en CO2e (MINAM, 2009). Para la conversión del carbono capturado en la estructura de la especie Phoenix dactylifera se utilizó el concepto de Certificados de Reducción de Emisiones (CRE's), donde un CRE corresponde a una tonelada métrica de dióxido de carbono equivalente (CO2e).

\section{RESULTADOS}

Para el cálculo de carbono los valores encontrados se multiplican por un factor de conversión 
para encontrar el carbono presente en la biomasa, el cual está en un rango de 45 a $50 \%$ de biomasa en las especies arbóreas. Para esta investigación se tomó el valor de 50 \% (factor 0,5). Finalmente, se obtuvo el CO2e multiplicando la cantidad o porcentaje de carbono con el factor de conversión producto de la relación de Peso Molecular existente ente ellos (Tabla 01).

Tabla 1. Cantidad de $\mathrm{CO}_{2}$ e en $\mathrm{Tn}$ y $\mathrm{Tn} / \mathrm{ha}$

\begin{tabular}{crrrrrrr}
\hline ZONA & $\begin{array}{l}\text { Tn C/ha } \\
\text { (Octubre) }\end{array}$ & $\begin{array}{c}\text { Tn C/ha } \\
\text { (Junio) }\end{array}$ & $\begin{array}{c}\text { CANTIDAD } \\
\text { de PCC }\end{array}$ & Fac & $\begin{array}{c}\text { Tn CO2e } \\
\text { /ha }\end{array}$ & $\begin{array}{l}\text { ÁREA TOTAL } \\
\text { (ha) }\end{array}$ & Tn CO2e \\
\hline 1 & 1,480 & 1,405 & 0,074 & 3,67 & 0,273 & 500 & 136,87 \\
2 & 0,256 & 0,246 & 0,009 & 3,67 & 0,036 & 500 & 18,34 \\
TOTAL & & & & & 0,310 & & 155,22 \\
\hline
\end{tabular}

La muestra Total de 69 palmeras distribuidas en las parcelas permanentes de $20 \mathrm{~m} \times 5 \mathrm{~m}$ que fueron ubicadas en cada uno de los 8 puntos por zona. Durante el tiempo de investigación, se registró un total de biomasa de $2,81 \mathrm{Tn} / \mathrm{ha}$ en el mes de junio, luego en octubre registró una biomasa de 2,96 Tn/ha para la zona 1, mientras para la zona 2 se registró una biomasa inicial (junio) de 0,49 Tn/ha y para el mes de octubre de 0,51 Tn/ha. Se calculó la cantidad de CO2e capturado durante los 4 meses que duró la investigación por parte de la biomasa arbórea aérea la cual fue de 0,27 Tn CO2e /ha para la zona 1 y 0,037 Tn CO2/ha para la zona 2.

Tabla 2. cuantificación mediante la biomasa arbórea aérea.

\begin{tabular}{lllll}
\hline & INTERVALO & BIOMASA & Fac & Tn C/ha \\
& (2 MESES) & Tn/ha & & \\
\hline \multirow{2}{*}{ ZONA 1 } & JUNIO & 2,81097307 & 0,5 & 1,40548654 \\
\multirow{2}{*}{ TOTAL PCC } & AGOSTO & 2,91622446 & 0,5 & 1,45811223 \\
& OCTUBRE & 2,96015885 & 0,5 & 1,48007942 \\
ZONA 2 & JUNIO & 0,49279193 & 0,5 & 0,24639597 \\
& AGOSTO & 0,50726148 & 0,5 & 0,25363074 \\
TOTAL PCC & OCTUBRE & 0,51278689 & 0,5 & 0,25639345 \\
\hline
\end{tabular}

Tabla 3. Captura de $\mathrm{CO}_{2} \mathrm{e}$ por la Phoenix dactylifera.

\begin{tabular}{cccc}
\hline ZONA & Tn CO2e /ha ÁREA TOTAL & $\begin{array}{c}\text { Tn CO2e } \\
\text { (ha) }\end{array}$ & \\
\hline 1 & 0,2737559 & 500 & 136,87795 \\
2 & 0,036690748 & 500 & 18,345374 \\
TOTAL & 0,310446648 & & 155,223324 \\
\hline
\end{tabular}

Se realizó la Correlación del carbono capturado por la Phoenix dactylifera con su crecimiento producto de las emisiones de $\mathrm{CO} 2$ del parque automotor en el distrito de Tacna. Se muestra 
que la correlación es directa y muy fuerte entre el carbono durante los meses muestreados, donde la relación entre el carbono obtenido en Junio respecto a Agosto ( 2 meses después) es de 0,9998 , la relación del carbono obtenido en Agosto respecto a Octubre ( 2 meses después) fue de 0,99998 para finalmente realizar la relación del carbono obtenido en el mes de Junio con el mes de Octubre (fecha de inicio y final de la investigación) dando 0,9998. El coeficiente de correlación se repite con los valores de $\mathrm{CO} 2$ e. Los valores dados entre las distintas Tn C y Tn CO2e del mismo mes dan 1 debido a que mantienen una relación de dependencia funcional. La relación más fuerte es de 0,99998 perteneciente entre el mes de Agosto con el mes de Octubre.

Se muestra que la correlación es directa y muy fuerte entre la obtención de carbono a lo largo de los meses muestreados, donde la relación entre el carbono obtenido en Junio respecto a Agosto es de 0,999999669, la relación del carbono obtenido en Agosto respecto a Octubre fue de 0,999999857 para finalmente realizar la relación del carbono obtenido en el mes de Junio con el mes de Octubre dando 0,999999875. El coeficiente de correlación se repite con los valores de CO2e. La relación más fuerte es de 0,999999875 perteneciente entre el mes de Junio con el mes de Octubre

El valor de correlación de Pearson es 0,9969 para las variables Altura y CO2e siendo una relación muy fuerte y directa, para el caso de Temperatura y CO2e la correlación es de 0,4999 mostrando una relación directa moderada, finalmente la Temperatura y la altura el valor de su correlación fue de 0,4304 mostrando también una relación directa moderada. El valor de Altura vs $\mathrm{CO}_{2}$ e es significativo porque ese valor tiene un $\mathrm{P}$-valor $\leq 0,05$.

Tabla 7. Resultado de correlación de Pearson del estrato II

\begin{tabular}{lccc}
\hline & ALTURA $(\mathrm{m})$ & $\mathrm{CO}_{2} \mathrm{e}(\mathrm{Tn})$ & TEMP. $\left({ }^{\circ} \mathrm{C}\right)$ \\
\hline ALTURA $(\mathrm{m})$ & & 1,000 & 0,473 \\
& & $(3)$ & $(3)$ \\
& & 0,0004 & 0,69 \\
$\mathrm{CO}_{2} \mathrm{e}(\mathrm{Tn})$ & 1,000 & & 0,4725 \\
& $(3)$ & $(3)$ \\
TEMP. $\left({ }^{\circ} \mathrm{C}\right)$ & 0,0004 & 0,69 \\
& 0,473 & 0,4725 & \\
& $(3)$ & $(3)$ & \\
\hline
\end{tabular}

El valor de correlación de Pearson es 1,000 para las variables Altura y CO2e siendo una relación muy fuerte y directa, para el caso de Temperatura y CO2e la correlación es de 0,4725 mostrando una relación directa moderada, finalmente la Temperatura y la altura el valor de su correlación fue de 0,4730 mostrando también una relación directa moderada. El valor de Altura vs $\mathrm{CO}$ e es significativo porque ese valor tiene un P-valor $\leq 0,05$.

\section{DISCUSIÓN}

Las palmeras Phoenix dactylifera distribuidas de forma heterogénea en las áreas verdes del 
distrito de Tacna, presenta una cantidad de 1006 individuos. Esta cifra se incrementó en comparación al último informe realizado por parte de la Municipalidad Provincial de Tacna (2012) donde se registró un total de 995 individuos sin embargo son escasos los trabajos relacionados a cuantificación de biomasa para la especie Phoenix dactylifera a nivel local y nacional. No obstante, en países como España se ha analizado a esta especie teniendo el enfoque paisajístico como Gracia (2006) donde se establece un censo de 181138 individuos con altura promedio de 9,4 $\mathrm{m}$ de estipe y diámetro de estipe de 0,42 $\mathrm{m}$ el cual difiere a las mediciones realizadas debido al enfoque mencionado y a los métodos aplicados sin especificar si incluye o no medición de tablas. Actualmente, la antigüedad de la Phoenix dactylifera tampoco es homogénea en los puntos descritos, la edad de esta especie es mayor en el corazón del distrito (el paseo cívico de la ciudad) y en zonas emblemáticas como la plaza MacLean y la plaza Cristóbal Colon con una edad aproximada no menor a 90 años, además la población más joven de esta especie se encuentran ubicadas en la avenida Industrial con una altura no mayor de $3 \mathrm{~m}$. y con una edad no menor a 30 años, con lo cual mantiene relación con lo descrito Gonzáles (2001), donde el desarrollo de la especie es inversa a su edad reduciéndose a medida que alcanza la edad adulta. Por otro lado, la cantidad total de carbono almacenado y carbono capturado por la Phoenix dactylifera mediante el aumento de su biomasa fue de 1,652 Tn C /ha almacenado para el mes de junio, 1,712 Tn C/ha almacenado para el mes de agosto y 1,736 Tn C/ha almacenado para el mes de octubre. El carbono capturado mediante la biomasa arbórea aérea fue de $0,084 \mathrm{Tn} \mathrm{C} /$ ha, resultante de la diferencia entre el carbono almacenado del mes de octubre con el carbono almacenado del mes de junio.

Asimismo, la cantidad de emisiones de $\mathrm{CO} 2$ capturado por parte de la Phoenix dactylifera fue de 155,223 Tn CO2e para las 2 zonas durante el mes de junio hasta octubre del 2016 que en comparación a los trabajos realizados por Castro \& Escobar (2006) y Londoño et al. (2011) no se utilizaron factores de emisión, conteo de vehículos en circulación, entre otros factores, para cuantificar $\mathrm{CO} 2$ en áreas de incidencia del parque automotor fuente constante de CO2. La comparación de resultados con las metodologías exclusivas de cantidad de dióxido de carbono por vehículo con el servicio ambiental de captura de carbono por especies arbóreas dará mayores aportes a fin de conocer las condiciones del distrito de Tacna.

La correlación de carbono capturado con su crecimiento producto de las emisiones de CO2 tiene según los resultados una relación directa muy fuerte, mostrando el desarrollo de la misma a través del tiempo, además, tanto Gracia (2006) como Navarro et al. (2013) coinciden en que el desarrollo de la Phoenix dactylifera se ve reflejado en su altura alcanzando en promedio de 0,10 m por año. Para Arana et al. (2014) la palma datilera se ha convertido a lo largo de los cuatro siglos de su introducción en una parte importante del ecosistema del desierto costero y de la cultura de sus pobladores (especialmente en Zaña y Ocuaje) debido a sus datos poblacionales que muestran estabilidad debido a una exitosa reproducción, en Tacna de igual manera es parte importante del sistema urbano debido a su estabilidad poblacional y por el servicio ambiental de captura de carbono descrito.

En efecto, la relación de carbono en biomasa con la cantidad de $\mathrm{CO} 2$ utilizada en esta investigación es corroborada por Navarro et al. (2013) y Ministerio del Ambiente (2009) donde se usa la relación de pesos moleculares entre el $\mathrm{CO} 2$ y C el cual está dado por un cofactor CF = 
3,67 y en consecuencia, el servicio ambiental predominante es la captura de carbono que coincide con los puntos con mayor cantidad de palmeras y fueron: Av. Bolognesi, Alameda Bolognesi, Av. Industrial y el tramo desde el Ovalo Callao hasta Ovalo Tarapacá representando el $86.1 \%$ del total de palmeras del distrito.

La capacidad de captura de carbono de la Phoenix dactylifera como servicio ambiental en el distrito de Tacna entendiéndose que (para fines de la investigación) el CO2 estuvo ligado a la condición de la calidad del aire, como condición fundamental para el desarrollo de la vida de las plantas (a través del aumento de biomasa) y a la cantidad de individuos de Phoenix dactylifera, debido a su prevalencia sobre las demás especies forestales del distrito de Tacna. El carbono almacenado por parte de la Phoenix dactylifera de las avenidas Bolognesi, avenida Industrial y alrededores para el mes de Junio fue 1,652 Tn C/ha, en Agosto de 1,712 Tn C/ha y Setiembre con 1,736 Tn C/ha. El carbono capturado mediante la biomasa arbórea aérea fue de $0,084 \mathrm{Tn}$ C/ha y se estimó las emisiones de $\mathrm{CO} 2$ capturado por parte de la Phoenix dactylifera obteniendo la cantidad de 155,223 Tn CO2e para las 2 zonas. La correlación que existe entre el carbono almacenado por la Phoenix dactylifera y el CO2e proveniente del parque automotor, concluyéndose que la relación es fuerte y directa $(+0.99)$ indicando que a mayor almacenamiento de carbono por parte de la especie mayor $\mathrm{CO} 2$ capturado por lo tanto menor cantidad de $\mathrm{CO} 2$ en el ambiente en el intervalo de tiempo que duró la investigación.

Se recomienda promover investigación para la generación de línea base a través de monitoreos biológicos por año de los datos dasométricos de Phoenix dactylifera, para determinar de manera más profunda la captura de carbono anual, así como estudios relacionados a servicios ambientales. De esta forma, se promovería de manera objetiva el cambio de actitud con respecto al medio ambiente. Realizar una guía de estimación de emisiones vehiculares, basándose en casos particulares (distritos) y en horas de concurrencia vehicular para que de esta manera sea más accesible, a fin de contar con otra fuente de información de presunta contaminación ambiental en determinado lugar o cuenca atmosférica.

\section{REFERENCIAS BIBLIOGRÁFICAS}

Arana, C., Salinas, L., Kahn, F., \& Millán, B. (2015). Impacto del Cambio Climático Global sobre la distribución geográfica de la Palma Datilera (Phoenix Dactylifera L.) en el Perú. R. Evento Científico. Lima, Perú: Vicerectorado de Investigación UNMSM.

Arana, C., Salinas, L., Millán, B., \& Kahn, F. (2014). Historia Natural de la Palma Datilera (Phoenix Dactylifera L.) en las principales localidades del Perú. R. Evento Científico. Lima, Perú: Vicerectorado de Investigación de la UNMSM.

Aranibar, L. F. (2014). Estado actual del bosque de Polylepis y su eficiencia en la captura de C02 en la provincia de Tarata, Departamento de Tacna. Tarata, Peru: Escuela de Postgrado de la UNJBG.

Balslev, H., Grandez, C., Paniagua Zambrana, N., \& Louise, A. (Noviembre de 2008). Palmas (Arecaceae) útiles en los alrededores de Iquitos, Amazonía Peruana. (F. d. UNMSM, Ed.) Peru Biol., 121-132.

Cabrera, H., \& Wallace, R. (2007). Densidad y Distribución espacial de palmeras arborescentes 
en un bosque preandino-amazónico de Bolivia. Ecología en Bolivia, 42, 121-135.

Canavos, C. G. (1988). Probabilidad y Estadística "Aplicaciones y Métodos". México: Mc GrawHill.

Cañizo, J. A. (2011). Palmeras Todos los géneros y 565 especies (3era ed.). España: MundiPrensa.

Carvajal Alcaraz, M., Mota, C., Alcaraz López, C., \& Iglesias, M. (2011). Investigación sobre la absorción de $\mathrm{CO} 2$ por los cultivos mas representativos. Consejo Superior de Investigaciones Científicas - Universidad de la Rioja, 58-63.

Carvajal, F. Z. (1969). Tacna, Historia y Folklore (2da ed.). Tacna, Perú: Santa María.

Castillo, M. C. (2014). Análisis del diseño de la política de la calidad del aire de la municipalidad metropolitana de Lima: 2010-2012. Tesis para optar el grado de Magister en Ciencia Política y Gobierno. Lima, Perú: Pontificia Universidad Católica del Perú.

Castro Peña, P. C., \& Escobar Winston, L. M. (2006). Estimación de las emisiones contaminantes por fuentes móviles a nivel nacional y formulación de lineamientos técnicos para el ajuste de las normas de emisión. Tesis de Grado. Bogotá D.C., Colombia.

Catpo, J. (2004). Determinación de la Ecuación alómetrica de Pinus patula y Estimación del contenido de carbono en su biomasa arbórea en Porcón, Cajamarca, Perú. Tesis para optar Título de Ingeniero Forestal. Cajamarca, Perú: UNALM.

CEZA, C. d. (Enero de 2010). Prospección y desarrollo de Germoplasma Varietal de Especies Frutales de Bajo requerimiento hídrico para aumentar la rentabilidad del Agua en el Norte Chico. Proyecto de inversión. Atacama - Coquimbo, Chile: Facultad de Ciencias Agronómicas - Universidad de Chile.

Cobos, A. D. (2012). Cuantificación de la Captura de Carbono en especies forestales y su contribución al ambiente en el Centro de Producción e Investigación Pabloyacu. Tesis de Grado. Moyobamba, Perú: UNSM-Tarapoto.

DGIAA. (2010). Mejoramiento del sistema de información para la Determinación de alertas atmosféricas en la ciudad de Tacna. Tacna: SENAMHI .

Diaz, J. A. (2011). Ingeniería Ambiental. MEXICO: ALFAOMEGA.

Dirección regional de salud Tacna. (2014). Gestión de la calidad del aire en la Ciudad de Tacna. Informe de Programa. Tacna, Perú: DIRESA.

Galeano, G., Pintaud, J.-C., Balslev, H., Bernal, R., Borchsenius, F., Ferreira, E., . . Kahn, F. (2008). Las palmeras en el marco de la investigación para el desarrollo en Ámerica del sur. Peru Biol., 5-6.

García, N. M. (Setiembre de 2011). Ajuste de Modelos de Captura de carbono para el tipo forestal Roble Raulí-Coigue y su Análisis Bioeconómico en la Reserva Nacional MallencoChile. Tesis Doctoral. Córdoba, España: Escuela Técnica Superior de Ingenieros Agrónomos y de Montes.

Gobierno de Canarias. (Enero de 2010). El picudo rojo de las pameras. Texto Institucional.

Canarias, España: Dirección general de Agricultura - Gobierno de Canarias.

Gobierno Regional de Tacna. (2014). Plan Basadre, Plan de Desarrollo Regional Desconcertado 2013-2023. Tacna: Consejo Regional de Tacna.

Gonzáles Pérez, M. (2001). Caracterización Molecular de la Palmera Canaria (Phoenix Canariensis) como base para su conservación. Tesis de Doctor. Las Palmas de Gran 
Canaria, España: DEPARTAMENTO DE BIOLOGÍA.

Gracerán, T. (2010). 30 Palmeras: Descripción, cuidados y cultivo. Fichas prácticas, 12-15.

México: De Vecchi.

Gracia i Vicente, L. (Abril de 2006). Indicadores Ambientales y Paisajísticos del Palmar del Elche. Tesis de Doctor. Valencia, España: Departamento de Agroquímica y Medio Ambiente.

GRT, G. R. (2014). Mejoramiento del Servicio de Monitoreo e Información Ambiental. Tacna: Gerencia regional de Recursos Naturales y Gestión del medio ambiente.

ICRAF, W. A. (2009). Guía para la determinación de carbono en pequeñas propiedades rurales. Manual Técnico. Lima, Perú.

INDECI. (2007). Programa de prevención y Medidas de Mitigación ante desastres de la ciudad de Tacna. Programa de Ciudades Sostenibles. Tacna: Instituto Nacional de Defensa Civil PNUD.

INEI. (26 de Agosto de 2016). Proyecciones departamentales de Población. Boletín. Perú: Instituto Nacional de Estadística e Informática.

IPCC. (2001). Tercer Informe de evaluación del grupo intergubernamental de Expertos sobre Cambio Climático. Reino Unido - Nueva York.

Kiely, G. (1999). INGENIERÍA AMBIENTAL Fundamentos, entornos, tecnologías y sistemas de gestión. España: Mc Graw Hill.

Laura Dawidowski, O. S. (2014). Estimaciones e emisiones vehiculares en Lima Metropolitana. Lima : SENAMHI.

Londoño, J., Correa, M., \& Palacio, C. (Diciembre de 2011). Estimación de las emisiones de contaminantes atmosféricos provenientes de fuentes móviles en el área urbana de Envigado, Colombia. Escuela de Ingeniería de Antioquia (16), 149-162.

MacDicken, K. G. (1997). A Guide to Monitoring Carbon storage in Forestry and Agroforestry projects. Winrock Internacional Institute.

Malca, A. G. (2001). Estimación de la capacidad de captura de CO2 en bosques secundarios del trópico Amazónico como indicador de Valorización Económica, Loreto-Perú.

Loreto: Instituto de Investigaciones de la Amazonía Peruana.

MINAM, M. d. (2009). Identificación de Metodologías existentes para determinar stock de carbono en ecosistemas forestales. Lima - Perú: Documento Institucional.

Morici, C. (2006). La Palmera Canaria: Phoenix canariensis. Revista Regional, 3, 134-143.

Canarias, España: Rincones del Atlántico.

Municipalidad Provincial de Tacna. (2012). Identificación y Cuantificación de Especies Forestales - Identificación de Plagas Comunes en los parques, plazas, óvalos y Áreas Verdes del distrito Tacna. Tacna: Proyecto.

Navarro, J., Díez Martín, B., \& Meléndez Pastor, I. (Setiembre de 2013). Estimación del contenido de $\mathrm{C}, \mathrm{N}$ y biomasa de El Palmeral de Elche (Patrimonio de la Humanidad). IndustriAmbiente, 50 - 54.

OPS, O. P. (2004). Guías para la Calidad del Aire. Lima: CEPIS, Centro Panamericano de Ingeniería Sanitaria y Ciencias del Ambiente.

Palomino, D. (2007). Estimación del servicio ambiental de captura del CO2 en la flora de los 
Humedales de Puerto Viejo. Tesis para optar el grado de Magister en Ciencias Ambientales. Lima, Perú: Universidad Nacional Mayor de San Marcos.

Pancca, P. C. (2014). Análisis del sistema de transporte público y la contaminación ambiental de los vehículos livianos en la ciudad de Tacna. Tesis de Postgrado. Tacna, Tacna, Peru: Universidad Privada de Tacna.

Pari Morales, Y. (2013). Estimación del carbono almacenado en la biomasa aérea

de plantaciones y rebrotes de eucalyptus globulus labill en la comunidad campesina de raquina - distrito de Pucará - Junín. Tesis para optar el Título de Ingeniero Forestal y Ambiental. Huancayo, Perú: Universidad Nacional del Centro del Perú.

Pari, A. (04 de Junio de 2009). Influencia del estado de los vehículos gasolineros y diesel de transporte masivo urbano en el proceso de inspección de gases contaminantes vehiculares en la ciudad de Tacna. Tesis para optar el grado de Maestro en Gestión Ambiental y Desarrollo Sostenible. Tacna, Tacna, Perú: Universidad Nacional Jorge Basadre Grohmann.

Reyes Avilés, I., \& Gutiérrez Chaparro, J. (2010 - 1). Los Servicios Ambientales de la Arborización Urbana: Retos y Aportes para la Sustentabilidad de la Ciudad de Toluca. Quivera, 96-103.

Ribas, R. S. (1999). Ingeniería Ambiental, Contaminación y Tratamientos. Barcelona: ALFAOMEGA.

Rivera Poma, J. (2012). Modelo de Identificación de factores contaminantes atmosféricos en Lima - Callao. Tesis para optar el grado de Magister en Ingeniería Industrial.

Lima, Perú: Universidad Nacional Mayor de San Marcos.

Rodríguez laguna, R., Jiménez Pérez, J., Meza Rangel, J., Aguirre Calderon, O., \& Razo Zarate, R. (Noviembre de 2007). Carbono contenido en un bosque tropical subcaducifolio en la reserva de la biosfera el cielo, Tamaulipas, México. Revista Latinoamericana de Recursos Naturales, 215.

Rojo, M., \& Figueroa, M. (2009). La Palmera, Símbolo de Poderío o Contribución al sistema verde en un Escenario de Cambio Climático. (U. I. Andalucía, Ed.)

Sancho, J. P. (2007). La poda de las palmeras, biología, ecología y gestión. Prácticas Culturas en Palmeras, (pág. 21). Canarias.

Sandra Fuentes Cubas, F. L. (2013). Evaluación de la captura de carbono en las especies forestales "Quinilla y Rupiña, en el centro de producción investigación Pabloyacu. Tesis de Grado. Moyobamba, Perú: UNSM.

Sobrino, G. M. (2008). Inducción de callos en Palma Datilera. Lima: UNALM.

UNFCCC, C. M. (2007). UNIDOS POR EL CLIMA. España: Ministerio de Medio Ambiente Español.

Valdés, T. V. (2005). Ecología y Medio Ambiente. MEXICO: PEARSON.

Valenzuela, G. C. (05 de Marzo de 2015). Valoración económica del servicio ambiental de captura de carbono en el Fundo Violeta, Distrito de Tahuamanu - Madre de Dios. Tesis para optar el Título de Licenciado en Geografía y Medio Ambiente. Lima, Perú: Pontificia Universidad Católica del Perú.

VALERA GARCIA, R. (2013). Determinación de la influencia de las condiciones climáticas en la captura de carbono en un sistema Theobroma sp "cacao" con sombra en Alto el SolPachiza -2012. Tesis para optar el Título de Ingeniero Ambiental. Moyobamba, Perú: Universidad Nacional de San Martín. 
Z. ABDELOUAHHAB. (2002). FAO CORPORATE DOCUMENT REPOSITORY. (FAO, Ed.) Recuperado el 15 de 11 de 2016, de http://www.fao.org/docrep/006

Zapata, M., del Valle, J. I., \& Orrego, S. A. (2009). Correcciones por sesgos en modelos Lognormales alometricos linealizados utilizados para estimación de la biomasa aérea. Publicación Universidad Nacional de Colombia. 